\title{
On Steady Flow of a Reactive Viscous Fluid in a Porous Cylindrical Pipe
}

\author{
Philip Iyiola Farayola \\ Department of Mathematics, Emmanuel Alayande College of Education, Oyo, Nigeria \\ Email:farayola_pi@yahoo.com
}

How to cite this paper: Farayola, P.I. (2017) On Steady Flow of a Reactive Viscous Fluid in a Porous Cylindrical Pipe. Open Journal of Fluid Dynamics, 7, 359-370. https://doi.org/10.4236/ojfd.2017.73024

Received: July 25, 2017

Accepted: September 19, 2017

Published: September 22, 2017

Copyright $\odot 2017$ by author and Scientific Research Publishing Inc. This work is licensed under the Creative Commons Attribution International License (CC BY 4.0).

http://creativecommons.org/licenses/by/4.0/

\begin{abstract}
Studies of mass transfer in a porous medium are of interest to researchers as a result of its various uses in different fields of engineering practices. This work examined the steady flow of a reactive variable viscous fluid in a porous cylindrical pipe. Dimensionless variables were used to dimensionalize the governing equations. A regular perturbation technique was employed to obtain an approximate solution of the resulting dimensionless non-linear equations. Numerical simulation was done to get the threshold values for the flow parameters under consideration. The effects of viscous heating and permeability parameters on the steady flow were studied and reported.
\end{abstract}

\section{Keywords}

Cylindrical Pipe, Viscosity, Permeability, Heating Parameter

\section{Introduction}

Fluid is a substance that yields readily to any force that alters its shape. Thus, it conforms to the configuration of a containing vessel. In physics, a fluid is a substance that continually deforms (flows) under an applied shear stress [1]. A reactive fluid, therefore, is any fluid that alters the internal geometry of a reservoir's porosity. In fluid dynamics, different reactive flows have been modeled generally by extending the Navier-Stokes equations to include the appropriate chemical reactions and forces. Viscosity is the property of a fluid that tends to prevent it from flowing when subjected to an applied force. High-viscosity fluids resist flow while low-viscosity fluids flow easily.

Pipe, in technology, is a tube (usually cylindrical) used to transport liquids, liquid-solid mixtures, or fragmented solids from one point to another. In petrochemical industries and petroleum refineries, studies related to thermal ignition 
and heat transfer in a reactive variable viscosity fluid are extremely useful in order to ensure safety of lives and properties [2] [3]. Thermal ignition occurs when a reaction produces heat too rapidly for a stable balance between heat production and heat loss to be preserved [4] [5] [6].

The study of flow of viscous fluid with temperature dependent properties is of great importance in lubrication and tribology (study of interacting moving surfaces, i.e. the science and technology of interacting surfaces in relative motion, including the study of friction, lubrication and wear), food processing, instrumentation and viscometry. However, viscous heating is always a possible and frequently significant source of errors in viscometric measurement at high shear rates in instrumentation and viscometry [7].

Many fluids used in industrial and engineering processes like geological materials, liquid foams, polymeric fluids, hydrocarbon oils and grease do exhibit flow characteristics that cannot be adequately described by the classical linearly viscous fluid model [8]. Ever since the pioneering work of Darcy on flow through porous beds which resulted in the identification of permeability as the property of porous media, there has been tremendous interest in the study of flow through porous media which occur widely in nature and industry [9].

Several researchers have worked on reactive variable viscosity flow with different non-porous channels [7] [8] [10] [11] [12] [13] [14] and porous channels [15] [16] [17] [18] [19] arriving at various results.

The objective of this paper is to study the steady flow of a reactive variable viscous fluid in a porous cylindrical pipe with an isothermal wall under Arrhenius kinetics and to report the effect of porosity and heating parameters on velocity and temperature of the flow.

\section{Mathematical Formulation of the Problem}

The work on steady flow of a reactive variable viscosity fluid in a cylindrical pipe with an isothermal wall under Arrhenius kinetics [12] was extended to include porosity force (permeability) in the momentum equation. The respective dimensional form of the energy and momentum equations with the boundary conditions are given as

$$
\begin{gathered}
\frac{1}{\bar{r}} \frac{\mathrm{d}}{\mathrm{d} \bar{r}}\left(\bar{r} \frac{\mathrm{d} T}{\mathrm{~d} \bar{r}}\right)+\frac{Q C_{0} A}{k} \mathrm{e}^{-\frac{E}{R T}}+\frac{\mu}{k}\left(\frac{\mathrm{d} \bar{u}}{\mathrm{~d} \bar{r}}\right)^{2}=0 \\
\frac{1}{\bar{r}} \frac{\mathrm{d}}{\mathrm{d} \bar{r}}\left(\mu \bar{r} \frac{\mathrm{d} \bar{u}}{\mathrm{~d} r}\right)+\frac{\mu}{k_{1}} \bar{u}=-G \\
\bar{u}=0, T=T_{0}, \quad \text { on } \bar{r}=a \\
\frac{\mathrm{d} T}{\mathrm{~d} \bar{r}}=\frac{\mathrm{d} \bar{u}}{\mathrm{~d} \bar{r}}=0, \quad \text { on } \bar{r}=0
\end{gathered}
$$

where $\mu_{0}, Q, C_{0}, A, E, R, T, G$ are the fluid reference viscosity, heat of reaction, initial concentration of the reactant species, rate of constant, activation energy, universal gas constant, absolute temperature and constant axial 
pressure gradient respectively, the last term $\frac{\mu}{k_{1}} \bar{u}$ in the left hand side of Equation (2) is the porosity force, $k_{1}$ is the Darcy permeability [15] [20] and $k$ is the thermal conductivity. The viscosity of the fluid is of the Arrhenius form given as

$$
\mu=\mu_{0} \mathrm{e}^{\frac{E}{R T}}
$$

where $\mu_{0}, E, R, T$ are as defined. Figure 1 showed the geometry of the steady flow in a cylindrical pipe. The fluid is assumed to be viscous, incompressible and reactive, flowing steadily in the z-direction with $\bar{r}=a$, and $\bar{u}=0$ at the wall. The temperature of the cylindrical wall is also assumed to be constant i.e. $T=T_{0}$ (isothermal) throughout the flow.

Introducing the following dimensionless quantities into Equations (1)-(4),

$$
\begin{aligned}
& r=\frac{\bar{r}}{a}, \quad u=\frac{\bar{u}}{b}, \quad \theta=\frac{E}{R T_{0}^{2}}\left(T-T_{0}\right), \\
& \epsilon=\frac{R T_{0}}{E}, \quad \mathrm{e}^{-\frac{E}{R T}}=\mathrm{e}^{\frac{\theta}{1+\epsilon \theta}} \mathrm{e}^{-\frac{1}{\epsilon}}, \quad \lambda=\frac{a^{2} Q C_{0} A}{k \in T_{0}} \mathrm{e}^{-\frac{1}{\epsilon}}, \\
& \beta=\frac{G^{2} a^{2}}{\mu_{0} Q C_{0} A}, \quad b=\frac{G a^{2}}{\mu_{0} \mathrm{e}^{\frac{1}{\epsilon}}}
\end{aligned}
$$

the dimensionless form of the governing equations are obtained as:

$$
\begin{gathered}
\frac{1}{r} \frac{\mathrm{d}}{\mathrm{d} r}\left(r \frac{\mathrm{d} \theta}{\mathrm{d} r}\right)+\lambda \mathrm{e}^{\frac{\theta}{1+\epsilon \theta}}+\lambda \beta \mathrm{e}^{\frac{-\theta}{1+\epsilon \theta}}\left(\frac{\mathrm{d} u}{\mathrm{~d} r}\right)^{2}=0 \\
\frac{1}{r} \frac{\mathrm{d}}{\mathrm{d} r}\left(r \mathrm{e}^{\frac{-\theta}{1+\epsilon \theta}} \frac{\mathrm{d} u}{\mathrm{~d} r}\right)+\delta \mathrm{e}^{\frac{-\theta}{1+\epsilon \theta}} u=-1
\end{gathered}
$$

with dimensionless boundary condition

$$
u(1)=\theta(1)=0, \frac{\mathrm{d} u(0)}{\mathrm{d} r}=\frac{\mathrm{d} \theta(0)}{\mathrm{d} r}=0
$$

where $\delta=\frac{a^{2}}{k_{1}}$ is the dimensionless porosity (permeability) parameter.

For all fuels of interest, the parameter $\in$ is assumed small [2] [12] [21] [22]. Using activation energy asymptotics and for $\in \ll 1$, Equations (5) and (6) are simplified to give

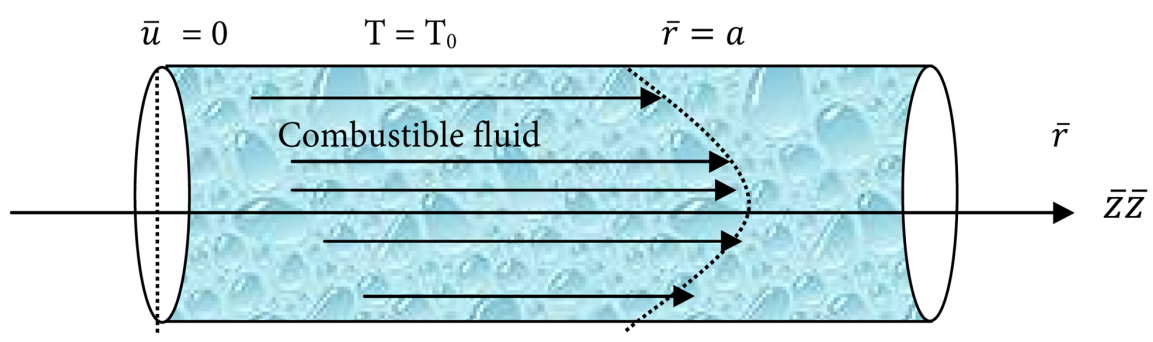

Figure 1. Geometry of the steady flow. 


$$
\begin{gathered}
\frac{1}{r} \frac{\mathrm{d}}{\mathrm{d} r}\left(r \frac{\mathrm{d} \theta}{\mathrm{d} r}\right)+\lambda \mathrm{e}^{\theta}+\lambda \beta \mathrm{e}^{-\theta}\left(\frac{\mathrm{d} u}{\mathrm{~d} r}\right)^{2}=0 \\
\frac{1}{r} \frac{\mathrm{d}}{\mathrm{d} r}\left(r \mathrm{e}^{-\theta} \frac{\mathrm{d} u}{\mathrm{~d} r}\right)+\delta \mathrm{e}^{-\theta} u=-1
\end{gathered}
$$

where Equations (8) and (9) represent the temperature and velocity equations respectively.

\section{Method of Solution}

The coupled nonlinear ordinary differential Equations (8) and (9), with the dimensionless boundary conditions (7), made it difficult to obtain exact solution. Since the cylindrical coordinate has singularity at $r=0$, singular perturbation technique was employed in order to obtain an approximate solution of the flow field and thermal distribution. It is convenient to take a power series expansion in the Frank-Kamenetskii parameter $\lambda$, and for easy computation, the solution to Equations (8) and (9) is assumed to be in the form

$$
u(r)=\sum_{i=1}^{\infty} \lambda^{i} u_{i}=u_{0}(r)+\lambda u_{1}(r)+\lambda^{2} u_{2}(r)+\lambda^{3} u_{3}(r)+\cdots
$$

and

$$
\theta(r)=\sum_{i=1}^{\infty} \lambda^{i} \theta_{i}=\theta_{0}(r)+\lambda \theta_{1}\left((r)+\lambda^{2} \theta_{2}(r)+\lambda^{3} \theta_{3}(r)+\cdots\right.
$$

Substituting Equations (10) and (11) into (8) and (9) using the dimensionless boundary conditions (7), we have

$$
\begin{aligned}
\theta(r)= & \lambda\left(\alpha_{4}+\alpha_{5} r^{2}+\alpha_{6} r^{4}+\alpha_{7} r^{6}+\alpha_{8} r^{8}\right)+\lambda^{2}\left(\alpha_{12}+\alpha_{13} r^{2}+\alpha_{14} r^{4}\right) \\
& +\lambda^{3}\left(\alpha_{18}+\alpha_{19} r^{2}+\alpha_{20} r^{4}\right)
\end{aligned}
$$

and

$$
\begin{aligned}
u(r)= & \alpha_{1}+\alpha_{2} r^{2}+\alpha_{3} r^{4}+\lambda\left(\alpha_{9}+\alpha_{10} r^{2}+\alpha_{11} r^{4}\right) \\
& +\lambda^{2}\left(\alpha_{15}+\alpha_{16} r^{2}+\alpha_{17} r^{4}\right)
\end{aligned}
$$

where

$$
\begin{gathered}
\alpha_{1}=-\frac{-16+\delta}{64-16 \delta+\delta^{2}} \\
\alpha_{2}=\frac{(-16+\delta) \delta}{4\left(64-16 \delta+\delta^{2}\right)}-\frac{1}{4} ; \\
\alpha_{3}=-\frac{(-16+\delta) \delta^{2}}{64\left(64-16 \delta+\delta^{2}\right)}+\frac{1}{64} \delta ; \\
\alpha_{4}=\frac{1}{4} \beta \alpha_{3}^{2}+\frac{4}{9} \alpha_{2} \alpha_{3} \beta+\frac{1}{4} \beta \alpha_{2}^{2}+\frac{1}{4} ; \\
\alpha_{5}=-\frac{1}{4}
\end{gathered}
$$




$$
\begin{aligned}
& \alpha_{6}=-\frac{1}{4} \beta \alpha_{2}^{2} \\
& \alpha_{7}=-\frac{4}{9} \alpha_{2} \alpha_{3} \beta \\
& \alpha_{8}=-\frac{1}{4} \alpha_{3}^{2} \beta \\
& \alpha_{9}=\frac{-16 \delta \alpha_{4} \alpha_{1}-64 \alpha_{4} \alpha_{2}-32 \alpha_{5} \alpha_{2}-64 \alpha_{4} \alpha_{3}-4 \delta \alpha_{5} \alpha_{1}+\delta^{2} \alpha_{4} \alpha_{1}}{64-16 \delta+\delta^{2}} \\
& \alpha_{10}=\frac{-\left(-16 \delta \alpha_{4} \alpha_{1}-64 \alpha_{4} \alpha_{2}-32 \alpha_{5} \alpha_{2}-64 \alpha_{4} \alpha_{3}-4 \delta \alpha_{5} \alpha_{1}+\delta^{2} \alpha_{4} \alpha_{1}\right) \delta}{4\left(64-16 \delta+\delta^{2}\right)} \\
& +\alpha_{4} \alpha_{2}+\frac{1}{4} \delta \alpha_{4} \alpha_{2} \\
& \alpha_{11}=\frac{\left(-16 \delta \alpha_{4} \alpha_{1}-64 \alpha_{4} \alpha_{2}-32 \alpha_{5} \alpha_{2}-64 \alpha_{4} \alpha_{3}-4 \delta \alpha_{5} \alpha_{1}+\delta^{2} \alpha_{4} \alpha_{1}\right) \delta^{2}}{64\left(64-16 \delta+\delta^{2}\right)} \\
& +\frac{1}{16} \delta \alpha_{4} \alpha_{2}+\frac{1}{2} \alpha_{5} \alpha_{2}+\alpha_{4} \alpha_{3}+\frac{1}{16} \delta \alpha_{5} \alpha_{1}-\frac{1}{64}\left(4 \alpha_{4} \alpha_{2}+\delta \alpha_{4} \alpha_{1}\right) \delta \\
& \alpha_{12}=\frac{1}{16\left(64-16 \delta+\delta^{2}\right)}\left(256 \alpha_{4}-64 \delta \alpha_{4}+4 \delta^{2} \alpha_{4}+8 \beta \delta^{2} \alpha_{2} \alpha_{1} \alpha_{5}+64 \alpha_{5}\right. \\
& -16 \delta \alpha_{5}+4 \beta \alpha_{2}^{2} \alpha_{4} \delta^{2}+256 \beta \alpha_{2}^{2} \alpha_{4}+\delta^{2} \alpha_{5}+64 \beta \delta \alpha_{2}^{2} \alpha_{4} \\
& \left.+128 \beta \delta \alpha_{2} \alpha_{3} \alpha_{4}+128 \beta \delta \alpha_{1} \alpha_{2} \alpha_{4}+64 \beta \delta \alpha_{2}^{2} \alpha_{5}\right) \\
& \alpha_{13}=\frac{-64 \alpha_{4}+16 \delta \alpha_{4}-\delta^{2} \alpha_{4}}{4\left(64-16 \delta+\delta^{2}\right)} \\
& \alpha_{14}=\frac{1}{16\left(64-16 \delta+\delta^{2}\right)}\left(-8 \beta \delta^{2} \alpha_{1} \alpha_{2} \alpha_{5}-64 \alpha_{5}+16 \delta \alpha_{5}-4 \beta \delta^{2} \alpha_{2}^{2} \alpha_{4}-256 \beta \alpha_{2}^{2} \alpha_{4}\right. \text {; } \\
& \left.-\delta^{2} \alpha_{5}-64 \beta \delta \alpha_{2}^{2} \alpha_{4}-128 \beta \delta \alpha_{2} \alpha_{3} \alpha_{4}-128 \beta \delta \alpha_{1} \alpha_{2} \alpha_{4}-64 \beta \alpha_{2}^{2} \delta \alpha_{5}\right) \\
& \alpha_{15}=\frac{1}{2\left(64-16 \delta+\delta^{2}\right)}\left(-\delta^{2} \alpha_{4}^{2} \alpha_{1}+2 \delta^{2} \alpha_{4} \alpha_{9}+2 \delta^{2} \alpha_{12} \alpha_{1}-128 \alpha_{4} \alpha_{10}-128 \alpha_{4} \alpha_{11}\right. \\
& -64 \alpha_{5} \alpha_{10}-32 \delta \alpha_{4} \alpha_{9}-8 \delta \alpha_{5} \alpha_{9}-32 \delta \alpha_{12} \alpha_{1}+16 \delta \alpha_{4}^{2} \alpha_{1}-128 \alpha_{12} \alpha_{2}+64 \alpha_{4}^{2} \alpha_{2} ; \\
& \left.+8 \delta \alpha_{5} \alpha_{4} \alpha_{1}-8 \delta \alpha_{13} \alpha_{1}+64 \alpha_{5} \alpha_{4} \alpha_{2}-64 \alpha_{13} \alpha_{2}-128 \alpha_{12} \alpha_{3}+64 \alpha_{4}^{2} \alpha_{3}\right) \\
& \alpha_{16}=\frac{-\delta}{8\left(64-16 \delta+\delta^{2}\right)}\left(-\delta^{2} \alpha_{4}^{2} \alpha_{1}+2 \delta^{2} \alpha_{4} \alpha_{9}+2 \delta^{2} \alpha_{12} \alpha_{1}-128 \alpha_{4} \alpha_{10}-128 \alpha_{4} \alpha_{11}\right. \\
& -64 \alpha_{5} \alpha_{10}-32 \delta \alpha_{4} \alpha_{9}-8 \delta \alpha_{5} \alpha_{9}-32 \delta \alpha_{12} \alpha_{1}+16 \delta \alpha_{4}^{2} \alpha_{1}-128 \alpha_{12} \alpha_{2}+64 \alpha_{4}^{2} \alpha_{2} \text {; } \\
& \left.+8 \delta \alpha_{5} \alpha_{4} \alpha_{1}-8 \delta \alpha_{13} \alpha_{1}+64 \alpha_{5} \alpha_{4} \alpha_{2}-64 \alpha_{13} \alpha_{2}-128 \alpha_{12} \alpha_{3}+64 \alpha_{4}^{2} \alpha_{3}\right) \\
& +\frac{1}{4} \delta \alpha_{4} \alpha_{9}+\alpha_{12} \alpha_{2}-\frac{1}{2} \alpha_{4}^{2} \alpha_{2}+\alpha_{4} \alpha_{10}+\frac{1}{4} \delta \alpha_{12} \alpha_{1}-\frac{1}{8} \delta \alpha_{4}^{2} \alpha_{1}
\end{aligned}
$$




$$
\begin{gathered}
\alpha_{17}=\frac{\delta^{2}}{128\left(64-16 \delta+\delta^{2}\right)}\left(-\delta^{2} \alpha_{4}^{2} \alpha_{1}+2 \delta^{2} \alpha_{4} \alpha_{9}+2 \delta^{2} \alpha_{12} \alpha_{1}-128 \alpha_{4} \alpha_{10}-128 \alpha_{4} \alpha_{11}\right. \\
-64 \alpha_{5} \alpha_{10}-32 \delta \alpha_{4} \alpha_{9}-8 \delta \alpha_{5} \alpha_{9}-32 \delta \alpha_{12} \alpha_{1}+16 \delta \alpha_{4}^{2} \alpha_{1}-128 \alpha_{12} \alpha_{2}+64 \alpha_{4}^{2} \alpha_{2} \\
\left.+8 \delta \alpha_{5} \alpha_{4} \alpha_{1}-8 \delta \alpha_{13} \alpha_{1}+64 \alpha_{5} \alpha_{4} \alpha_{2}-64 \alpha_{13} \alpha_{2}-128 \alpha_{12} \alpha_{3}+64 \alpha_{4}^{2} \alpha_{3}\right) \\
+\frac{1}{2} \alpha_{10} \alpha_{5}-\frac{1}{16} \delta \alpha_{5} \alpha_{4} \alpha_{1}-\frac{1}{2} \alpha_{5} \alpha_{4} \alpha_{2}-\frac{1}{32} \delta \alpha_{4}^{2} \alpha_{2}+\alpha_{4} \alpha_{11}-\frac{1}{2} \alpha_{4}^{2} \alpha_{3} \\
+\frac{1}{16} \delta \alpha_{13} \alpha_{1}+\frac{1}{16} \delta \alpha_{4} \alpha_{10}+\frac{1}{16} \delta \alpha_{12} \alpha_{2}+\frac{1}{2} \alpha_{13} \alpha_{2}+\frac{1}{16} \delta \alpha_{5} \alpha_{9} \\
+\alpha_{12} \alpha_{3}-\frac{1}{64}\left(\delta \alpha_{4} \alpha_{9}+4 \alpha_{12} \alpha_{2}-2 \alpha_{4}^{2} \alpha_{2}+4 \alpha_{4} \alpha_{10}+\delta \alpha_{12} \alpha_{1}-\frac{1}{2} \delta \alpha_{4}^{2} \alpha_{1}\right) \delta \\
\alpha_{18}=\frac{1}{8} \alpha_{4}^{2}+\frac{1}{4} \alpha_{12}+\frac{1}{16} \alpha_{5} \alpha_{4}+\frac{1}{8} \beta \alpha_{4}^{2} \alpha_{2}^{2}+\frac{1}{4} \beta \alpha_{10}^{2}+\frac{1}{16} \alpha_{13} \\
+\frac{1}{2} \beta \alpha_{2} \alpha_{16}-\frac{1}{2} \beta \alpha_{2} \alpha_{4} \alpha_{10}-\frac{1}{4} \beta \alpha_{12} \alpha_{2}^{2} \\
\alpha_{19}=-\frac{1}{8} \alpha_{4}^{2}-\frac{1}{4} \alpha_{12} \\
\alpha_{20}=-\frac{1}{2} \beta \alpha_{2} \alpha_{16}-\frac{1}{4} \beta \alpha_{10}^{2}-\frac{1}{8} \beta \alpha_{4}^{2} \alpha_{2}^{2}+\frac{1}{2} \beta \alpha_{2} \alpha_{4} \alpha_{10} \\
-\frac{1}{16} \alpha_{13}+\frac{1}{4} \beta \alpha_{12} \alpha_{2}^{2}-\frac{1}{16} \alpha_{14} \alpha_{5}
\end{gathered}
$$

\section{Results and Discussion}

Figure 2 showed the graph of $\theta(r)$ against $r$ for different values of heating

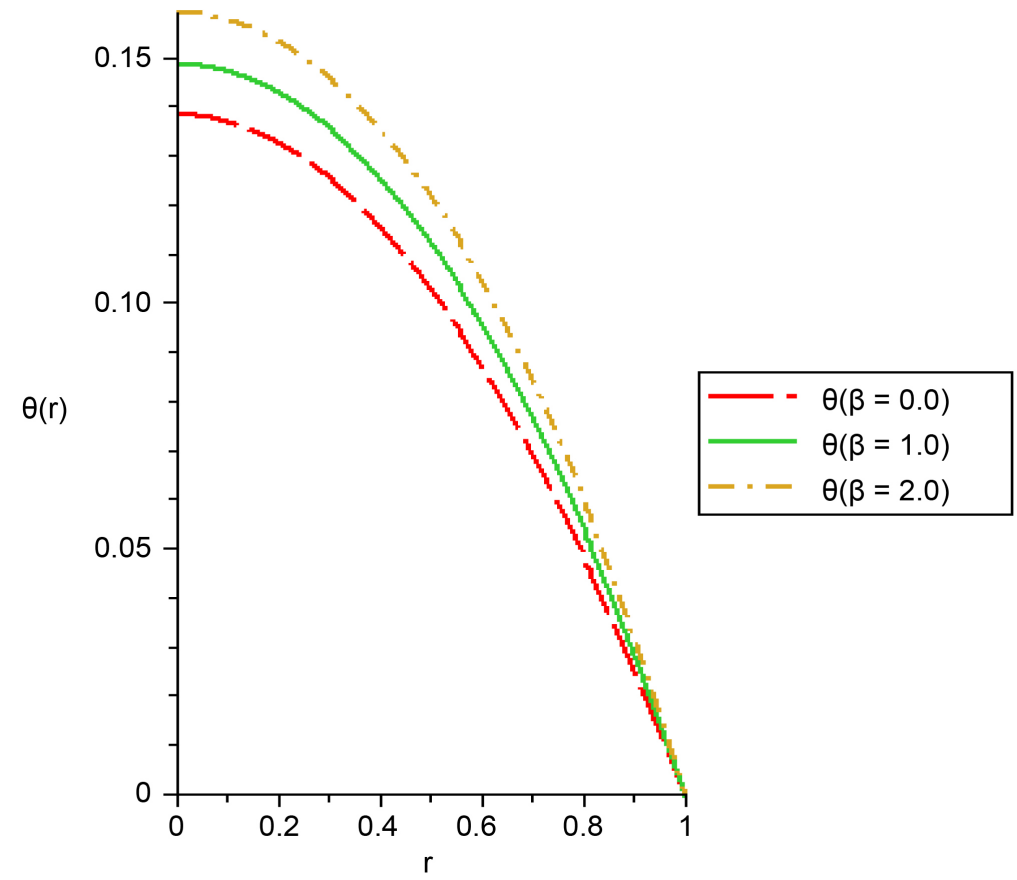

Figure 2. Plots of $\theta(r)$ against $r$ for different values of heating parameter $(\beta)$ when $\delta=0.0$ and $\lambda=0.5$. 
parameter $\beta$, when permeability parameter $\delta=0.0$ and the Frank-Kamenetskii parameter $\lambda=0.5$. Figure 3 showed the graph of $\theta(r)$ against $r$ for different values of heating parameter $\beta$ when $\delta=0.5$ and $\lambda=0.5$. It can be observed from Figure 2 that as the viscous heating parameter $\beta$ increases, the temperature also increases when the permeability parameter $\delta=0.0$ and the Frank-Kamenetskii parameter $\lambda=0.5$. This is in agreement with [13].

The maximum temperature appeared at the centre of the cylindrical pipe and it decreases towards the boundary of the pipe. This result is in line with [14] [22].

Figure 3 showed the temperature profile in the presence of permeability parameter $\delta=0.5$. The fluid temperature increases with increasing values of viscous heating parameter $\beta$. A critical comparison of Figure 2 and Figure 3 showed that in the presence of porosity parameter, the temperature of the fluid is a bit enhanced than when there is no porosity parameter. This implies that heat is added to the system as a result of the pores or voids in the flow.

Figure 4 depicted the graph of velocity $u(r)$ against $r$ for different values of $\beta$ when $\delta=0.0$ and $\lambda=0.5$. In Figure 4 , velocity increases with increasing values of the viscous heating parameter $\beta$ when $\lambda=0.5$ and permeability parameter $\delta=0.0$. This is in line with [17]'s work when there is no permeability parameter and $\lambda=0.5$.

Figure 5 is a graph of $u(r)$ against $r$ for different values of $\beta$ when $\delta=0.5$ and $\lambda=0.5$. Figure 6 showed the graph of $u(r)$ against $r$ for different values of permeability parameter $\delta$ when $\beta=0.0$ and $\lambda=0.5$ while

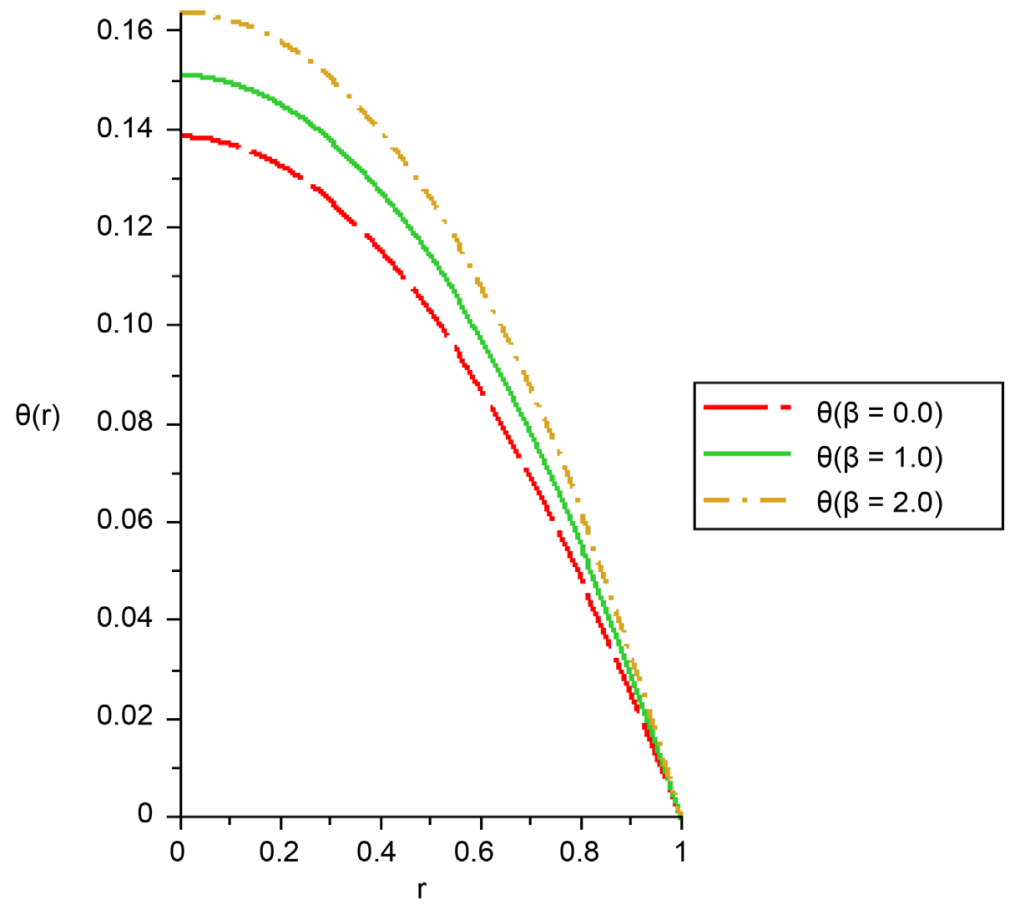

Figure 3. Plots of $\theta(r)$ against $r$ for different values of heating parameter ( $\beta$ ) when $\delta=0.5$ and $\lambda=0.5$. 


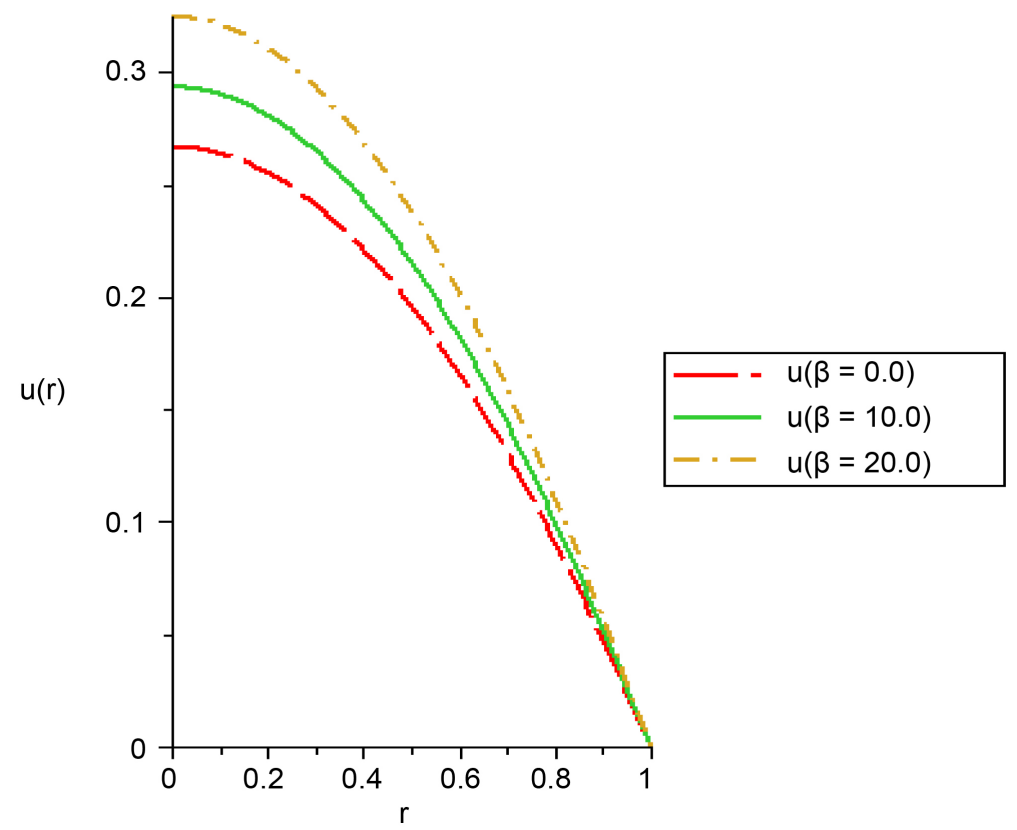

Figure 4. Plots of $u(r)$ against $r$ for different values of heating parameter $(\beta)$ when $\delta=0.0$ and $\lambda=0.5$.

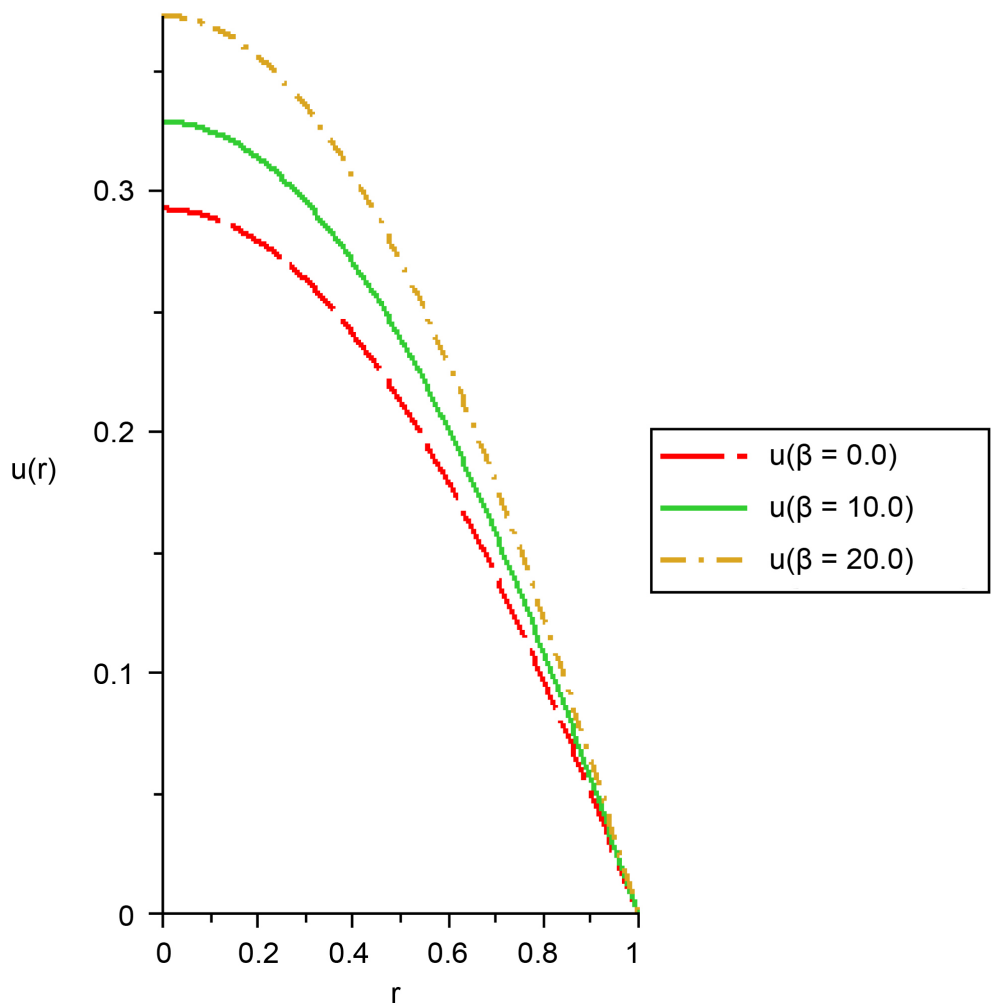

Figure 5. Plots of $u(r)$ against $r$ for different values of heating parameter $(\beta)$ when $\delta=0.5$ and $\lambda=0.5$.

Figure 7 showed the graph of $u(r)$ against $r$ for different values of $\delta$ when $\beta=10.0$ and $\lambda=0.5$. The graph in Figure 5 showed the velocity of the 


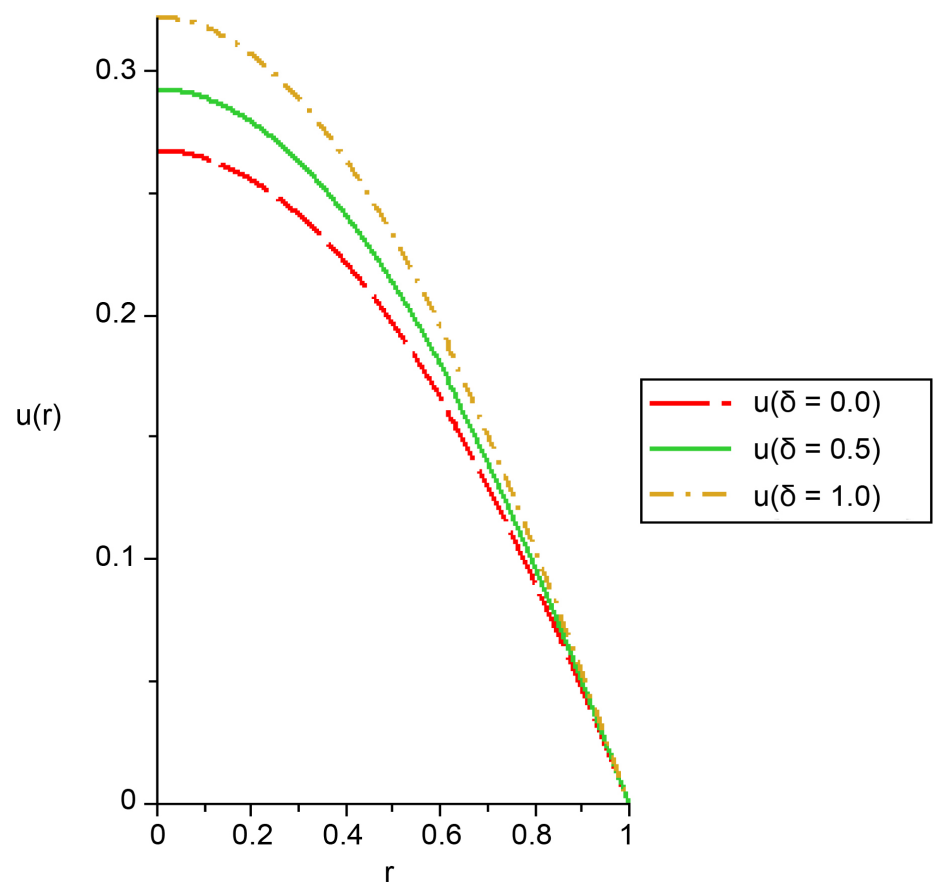

Figure 6. Plots of $u(r)$ against $r$ for different values of permeability parameter $(\delta)$ when $\beta=0.0$ and $\lambda=0.5$.

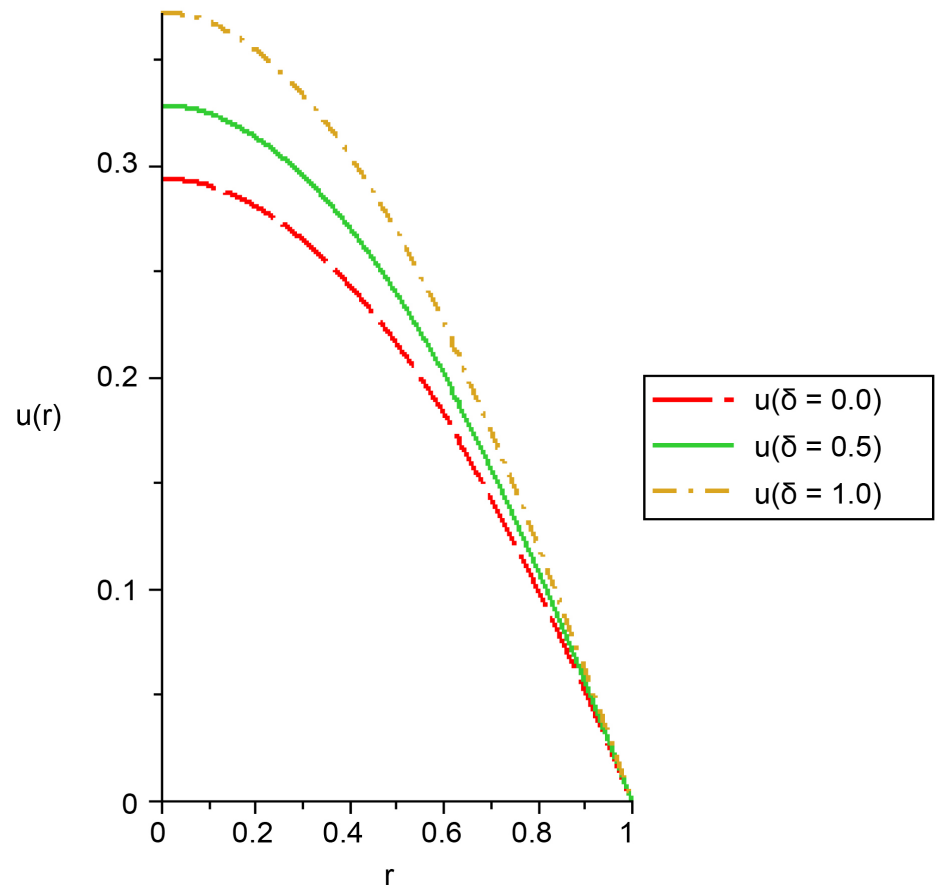

Figure 7. Plots of $u(r)$ against $r$ for different values of permeability parameter $(\delta)$ when $\beta=10.0$ and $\lambda=0.5$.

flow in the presence of permeability parameter. It is observed that velocity increases with increasing viscous heating parameter. It can be said that a combustible fluid at low viscosity flows faster than the one at high viscosity. A simi- 
lar thing is observed in the velocity profile for various values of the porosity parameter. Velocity increases with increasing values of permeability parameter. Permeability is the measure of the material's ability to permit liquid or gas through its pores or voids. Filters made of soil and earth dams are very much based upon the permeability of a saturated soil under load. Permeability is a part of the proportionality constant in Darcy's law. Darcy's law relates the flow rate and fluid properties to the pressure gradient applied to the porous medium. This supports that as permeability increases velocity should also increase [12].

\section{Conclusion}

From the findings of this work, it can be concluded that the viscous heating parameter has effect on the temperature and velocity profiles of the flow. The presence of pores on the geometry of the problem also has effect on the temperature and velocity of the fluid. Increase in permeability parameter increases the velocity of the fluid which is in agreement with the results of [12] [23]. The temperature is also enhanced than when there is no porosity. From the work, it could be said that the temperature of the fluid can be controlled by varying the viscous heating parameter and the thermal conductivity in order to minimize their effects and maximize the flow system efficiency.

\section{Applications}

Drilling an oil well can be very expensive. Therefore an exploration of a site for oil well must be economically feasible before embarking on it. Some of the factors to be considered for effective oil exploration are the porosity and permeability of the reservoir rock. In deciding the suitability of drilling an oil well, it is necessary that the porosity be a minimum of 8 per cent. The distance between the pores in the sandstone should not be too far apart as the oil and gas would not be able to flow well thereby resulting in poor outputs. In this paper, it has been shown that when sandstone have low permeability as indicated in Figure 6 and Figure 7, the flow velocity is affected and hence a large amount of pressure is required to push the oil and gas from the well. Therefore a successful oil well relies much on the right porosity and permeability of the sandstone. So, to ensure a good well, high permeability is required.

\section{Acknowledgements}

I appreciate the Tertiary Education Trust Fund (TETFund), Abuja, Nigeria for sponsoring me to attend the $10^{\text {th }}$ International Conference on Computational Heat, Mass and Momentum Transfer (ICCHM ${ }^{2} \mathrm{~T}$ 2017) organized by Korean Society for Fluid Machinery (KSFM) held at Sejong Hotel, Seoul, South Korea where the paper was first presented.

\section{References}

[1] Bird, R.B., Stewart, W.E and Lightfoot, E.N. (2002) Transport Phenomena. John 
Wiley \& Sons, New York.

[2] Bebernes, J. and Eberly, D. (1989) Mathematical Problems from Combustion Theory. Springer-Verlag, New York, 6-8.

https://doi.org/10.1007/978-1-4612-4546-9

[3] Bowes, P.C. (1984) Self-heating: Evaluating and Controlling the Hazard. Elsevier Sciences, Amsterdam.

[4] Balakrishman, E., Swift, A. and Wake, G.C. (1996) Critical Values for Some Non-Class Geometries in Thermal Ignition Theory. Mathematical and Computer Modelling, 24, 1-10.

[5] Makinde, O.D. (2004) Exothermic Explosions in a Slab: A Case Study of Series Summation Technique. International Communications in Heat and Mass Transfer, 31, 1227-1231.

[6] Makinde, O.D. (2005) Strong Exothermic Explosions in a Cylindrical Pipe: A Case Study of Series Summation Technique. Mechanics Research Communications, 32, 191-195.

[7] Adegbie, K.S. and Alao, F.T. (2007) Flow of Temperature-Dependent Viscous Fluid between Parallel Heated Walls: Exact Analytical Solutions in the Presence of Viscous Dissipation. Journal of Mathematics and Statistics, 3, 12-14. https://doi.org/10.3844/jmssp.2007.12.14

[8] Makinde, O.D. (2009) Thermal Criticality for a Reactive Gravity Driven Thin Film Flow of a Third-Grade Fluid with Adiabatic Free Surface down an Inclined Plane. Applied Mathematics and Mechanics, 30, 373-380. https://doi.org/10.1007/s10483-009-0311-6

[9] Basak, T., Kaluri, R.S. and Balakrishnan, A.R. (2012) Entropy Generation during Natural Convection in a Porous Cavity: Effect of Thermal Boundary Conditions. Numerical Heat Transfer, Part A, 62, 336-364. https://doi.org/10.1080/10407782.2012.691059

[10] Ajadi, S.O. (2010) Analytical Solutions of Unsteady Oscillatory Particulate Visco-Elastic Fluid between Two Parallel Walls. International Journal of Nonlinear Science, 9, 131-138.

[11] Attia, H.A. (2005) The Effect of Suction and Injection on the Steady Flow between Two Parallel Plates with Variable Properties. Tamkang Journal of Science and Engineering, 8, 17-22.

[12] Loganathan, P. and Sivapoornapriya, C. (2014) Viscous Dissipation Effects on Unsteady Natural Convective Flow Past an Infinite Vertical Plate with Uniform Heat and Mass Flux. Wseas Transactions on Heat and Mass Transfer. 9, 63-73.

[13] Makinde, O.D. (2007) On Steady Fow of a Reactive Variable Viscosity Fluid in a Cylindrical Pipe with an Isothermal Wall. International Journal of Numerical Methods for Heat and Fluid Flow, 17, 187-194. https://doi.org/10.1108/09615530710723957

[14] Pal, D. and Mondal, H. (2014) Effects of Temperature Dependent Viscosity and Variable Thermal Conductivity on MHD Non-Darcy Mixed Convective Diffusion of Species over a Stretching Sheet. Journal of the Egyptian Mathematical Society, 22, 123-133.

[15] Attia, H.A. (2009) Effect of Porosity on Unsteady Poiseuille Flow of a Viscoelastic Fluid with Temperature Dependent Viscosity. Tamkang Journal of Science and Engineering, 12, 215-221.

[16] Brinkman, H.C. (1947) On the Permeability of Media Consisting of Closely Packed 
Porous Particles. Applied Scientific Research, A1, 81-86.

[17] Chand, G. and Jat, R.N. (2014) Viscous Dissipation and Radiation Effects on MHD Flow and Heat Transfer over an Unsteady Stretching Surface in a Porous Medium. Thermal Energy and Power Engineering, 3, 266-272.

[18] Mohamed, R.A. (2009) Double-Diffusive Convection-Radiation Interaction on Unsteady MHD Flow over a Vertical Moving Porous Plate with Heat Generation and Soret Effect. Applied Mathematical Sciences, 3, 629-651.

[19] Uddin, M.S. (2013) Chemically Reactive Solute Transfer over a Plate in Porous Medium in Presence of Suction. Journal of Physical Sciences, 17, 97-109.

[20] Makinde, O.D. and Eegunjobi, A.S. (2013) Effects of Convective Heating on Entropy Generation Rate in a Channel with Permeable Walls. Entropy, 15, 220-233. https://doi.org/10.3390/e15010220

[21] Gbadeyan, J.A. and Hassan, A.R. (2012) Multiplicity of Solutions for a Reactive Variable Viscous Couette Flow under Arrhenius Kinetics. Mathematical Theory and Modeling, 2, 39-49.

[22] Olayiwola, R.O. (2012) Fixed-Bed Solid Fuel Arrhernius Combustion: Modelling and Simulation. Journal of the Nigerian Association of Mathematical Physics, 21, 111-120.

[23] Celestina, A.O. and Ayeni, R.O. (2010) On the Theory of Filtration at a Decreasing Rate. Journal of the Nigerian Association of Mathematical Physics, 16, 305-316.

\section{Submit or recommend next manuscript to SCIRP and we will provide best} service for you:

Accepting pre-submission inquiries through Email, Facebook, LinkedIn, Twitter, etc. A wide selection of journals (inclusive of 9 subjects, more than 200 journals)

Providing 24-hour high-quality service

User-friendly online submission system

Fair and swift peer-review system

Efficient typesetting and proofreading procedure

Display of the result of downloads and visits, as well as the number of cited articles

Maximum dissemination of your research work

Submit your manuscript at: http://papersubmission.scirp.org/

Or contact ojfd@scirp.org 